Proc. of the 15th Int. Workshop on Slow Positron Beam Techniques and Applications, Prague, September 2-6, 2019

\title{
Positron Structural Analysis of ScN Films Deposited on MgO Substrate
}

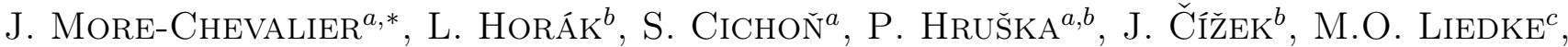 \\ M. Butterling ${ }^{c}$, A. WAgneR ${ }^{c}$, J. Bulír ${ }^{a}$, P. HubíK ${ }^{a}$, Z. Gedeonováa And J. LAnČOK ${ }^{a}$ \\ ${ }^{a}$ Institute of Physics of the Czech Academy of Sciences, Na Slovance 2, 18221 Praha 8, Czechia \\ ${ }^{b}$ Charles University, Faculty of Mathematics and Physics, V Holesovickach 2, 18000 Praha 8, Czechia \\ ${ }^{c}$ Helmholtz-Zentrum Dresden-Rossendorf, Institute of Radiation Physics, \\ Bautzner Landstr. 400, 01328 Dresden, Germany
}

\begin{abstract}
Scandium nitride $(\mathrm{ScN})$ is a semiconductor with a rocksalt-structure that has attracted attention for its potential applications in thermoelectric energy conversion devices, as a semiconducting component in epitaxial metal/semiconductor superlattices. Two ScN films of $118 \mathrm{~nm}$ and $950 \mathrm{~nm}$ thicknesses were deposited at the same conditions on $\mathrm{MgO}$ (001) substrate by reactive magnetron sputtering. Poly-orientation of films was observed with first an epitaxial growth on $\mathrm{MgO}$ and then a change in the orientation growth due to the decrease of the adatom mobility during the film growth. Positron lifetime measurements showed a high concentration of nitrogen vacancies in both films with a slightly higher concentration for the thicker ScN film. Presence of nitrogen vacancies explains the values of direct band gaps of $2.53 \pm 0.01 \mathrm{eV}$, and $2.56 \pm 0.01 \mathrm{eV}$ which has been measured on ScN films of $118 \mathrm{~nm}$ and $950 \mathrm{~nm}$ thicknesses, respectively.
\end{abstract}

DOI: 10.12693/APhysPolA.137.209

PACS/topics: $78.70 . \mathrm{Bj}, 41.75 . \mathrm{Fr}, 77.84 . \mathrm{Bw}$

\section{Introduction}

Scandium nitride is a transition metal nitride semiconductor with remarkable properties such as high hardness, high melting point, and high electron mobility [1-3]. This material has great potential for thermoelectric and piezoelectric device $[4,5]$. A wide range of techniques has been used to deposit $\mathrm{ScN}$ films like reactive evaporation, molecular beam epitaxy, reactive sputtering, and chemical vapor deposition $[2,3,6-8]$. Two directs band gaps in the range of $1.8-2.6 \mathrm{eV}$ and $2.9-4.9 \mathrm{eV}$ are calculated from the $X$ valence band to the $X$ conduction band, and from the $\Gamma$ valence band to the $\Gamma$ conduction band, respectively [9-11]. Experimental studies have reported the measurements of the first direct band gap in the range of $2.03-3.2 \mathrm{eV}$ and recently a measurement of the second direct band gap of $4.25 \mathrm{eV}[5,12]$. The difference between the experimental and calculated results have been attributed to structural defects, nitrogen vacancies, and impurities [7, 13]. Presence of impurities such as oxygen is clearly dependent on the base pressure of the chamber regardless on the used technique which has to be around $10^{-8} \mathrm{~Pa}$ in order to minimize these contaminations during the deposition. In the case of $\mathrm{ScN}$, concentration and type of defects depend on the deposition technique used in the film growth and several parameters have been tested and play important roles on the defect structure of films.

\footnotetext{
* corresponding author; e-mail: joris.more.chevalier@gmail.com
}

Hybrid vapor phase epitaxy (HVPE) method is a technique allowing to deposit $\mathrm{ScN}$ free from such defects. Dismukes et al. performed HVPE ScN deposition and they observed stoichiometric films for a series of films with no increase in donor concentration over the range of growth temperature from $750^{\circ} \mathrm{C}$ to $1150^{\circ} \mathrm{C}$ [3]. ScN deposited by molecular-beam epitaxy (MBE) showed that the $\mathrm{ScN}$ stoichiometry is strongly dependent on the $\mathrm{N} / \mathrm{Sc}$ flux ratio during the deposition process [14]. In the case of scandium rich conditions, the $\mathrm{N} / \mathrm{Sc}$ ratio approximately equaled the flux ratio during the growth process which resulted in nitrogen vacancies. For nitrogen rich conditions, nitrogen vacancies were suppressed and the films were stoichiometric. Similar results were reported for almost stoichiometric ScN films deposited via magnetron sputtering [15]. Deposition temperature is also an important parameter to ensure stoichiometric films via magnetron sputtering. $\mathrm{N} / \mathrm{Sc}$ ratios varying from 0.63 to 0.93 were observed for $\mathrm{ScN}$ films deposited on $\mathrm{MgO}$ (111) as a function of the deposition temperature from $700^{\circ} \mathrm{C}$ to $950^{\circ} \mathrm{C}[16]$.

In this work, a structural analysis of $\mathrm{ScN}$ films has been reported on two different film thicknesses. X-ray diffraction and positron annihilation spectroscopy measurements have been performed in order to analyze structural defects together with vacancies into $\mathrm{ScN}$ films deposited on $\mathrm{MgO}$ (001) substrates. Optical properties have been examined and a significant connection has been done between the direct band gap values and structural defects in the films. 


\section{Experimental}

The $\mathrm{ScN}$ layers were deposited on double-side polished $\left(10 \times 10 \times 0.5 \mathrm{~mm}^{3}\right) \mathrm{MgO}(001)$ substrate in an ultrahigh-vacuum (UHV) system (base pressure $\approx 10^{-8} \mathrm{~Pa}$ ) by $\mathrm{DC}$ reactive magnetron sputtering using a mixture $\mathrm{Ar}$ (99.99999\% pure) and $\mathrm{N}_{2}$ (99.9999\% pure) discharge with a ratio of $55 / 45$ respectively. The $\mathrm{MgO}$ substrates were cleaned using several steps, first with acetone in an ultrasonic bath, then repeated with methanol and blown dry with an $\mathrm{N}_{2}$-gun. The sample holder was electrically heated first at $300{ }^{\circ} \mathrm{C}$ in order to be degassed for $1 \mathrm{~h}$ and then increased until the film deposition temperature of $700^{\circ} \mathrm{C}$. Temperature was measured by two thermocouples attached to the sample holder. The working pressure of $2 \mathrm{~Pa}$ was used to sputter $\mathrm{ScN}$ from Sc target (NEYCO: Sc $99.9 \%$ ) of one-inch diameter with a detectable contaminant of Ta at $0.1 \%$. A sputtering power density of $2.8 \mathrm{Wcm}^{-2}$ was used to deposit films with two thicknesses of $118 \pm 10 \mathrm{~nm}$ and $950 \pm 30 \mathrm{~nm}$. The thicknesses were determined using a KLA Tencor profilometer and confirmed by spectroscopic ellipsometry.

$\mathrm{ScN}$ crystalline quality was characterized by X-ray diffraction (XRD) employing $\omega / 2 \theta$ scans, rocking curve ( $\omega$-scans), and pole figures measurements. A Panalytical MRD diffractometer with a $\mathrm{Cu}$ line-focus anode source $(\lambda=1.540593 \AA)$, a parabolic X-ray mirror, and a 2 -bounce monochromator Ge (220)x2 in the primary beam were used.

Positron lifetime studies were performed on a pulsed slow positron beam MePS with energy of incident positrons variable in the range from 1 to $16 \mathrm{keV}$ [17] corresponding to mean positron implantation depths into ScN from 9 to $795 \mathrm{~nm}$. Positron lifetime spectra were measured using a digital spectrometer with time resolution of $\approx 250 \mathrm{ps}$ (FWHM of resolution function), 10 millions positron events was collected in each spectrum.

Resistivity and the Hall effect measurements were carried out using the differential van der Pauw (vdP) method in a quasi-square arrangement at room temperature $298 \pm 1 \mathrm{~K}$, using a Keithley 6221 current source and two electrometers, a Keithley 6514 with a Keithley 2182A nanovoltmeter, which recorded the voltage difference between the electrometers together with a Keithley 708B switching matrix. The linearity of the contacts was checked for all measured samples in order to ensure contact ohmicity. A magnetic field of $\pm 0.20 \mathrm{~T}$ was applied for the Hall effect measurements.

The reflectance and transmittance spectra were acquired using a spectrophotometer Lambda 1050 (Perkin Elmer) in the range from $190 \mathrm{~nm}$ to $3200 \mathrm{~nm}$. The absorption coefficients were calculated from these results.

\section{Results and discussion}

Figure 1 shows the XRD $\omega / 2 \theta$ scans over the $2 \theta$ range between $5^{\circ}$ and $155^{\circ}$, for both ScN layers. Poly-oriented films are observed with preferential (002) and (111) grain

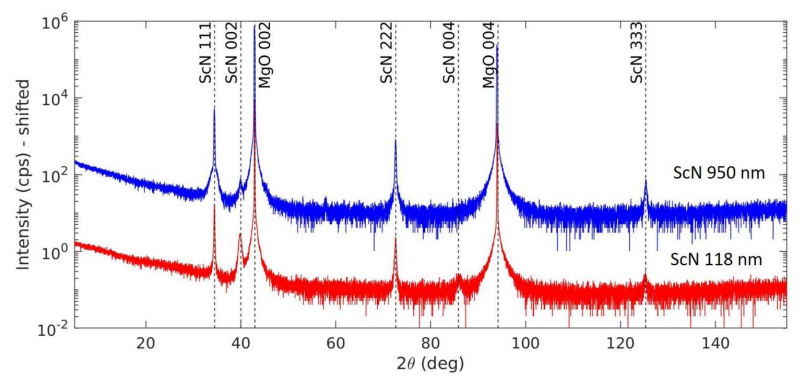

Fig. $1 . \omega / 2 \theta$ scans over the $2 \theta$ range $5-155^{\circ}$ of both ScN layers of $118 \mathrm{~nm}$ and $950 \mathrm{~nm}$.
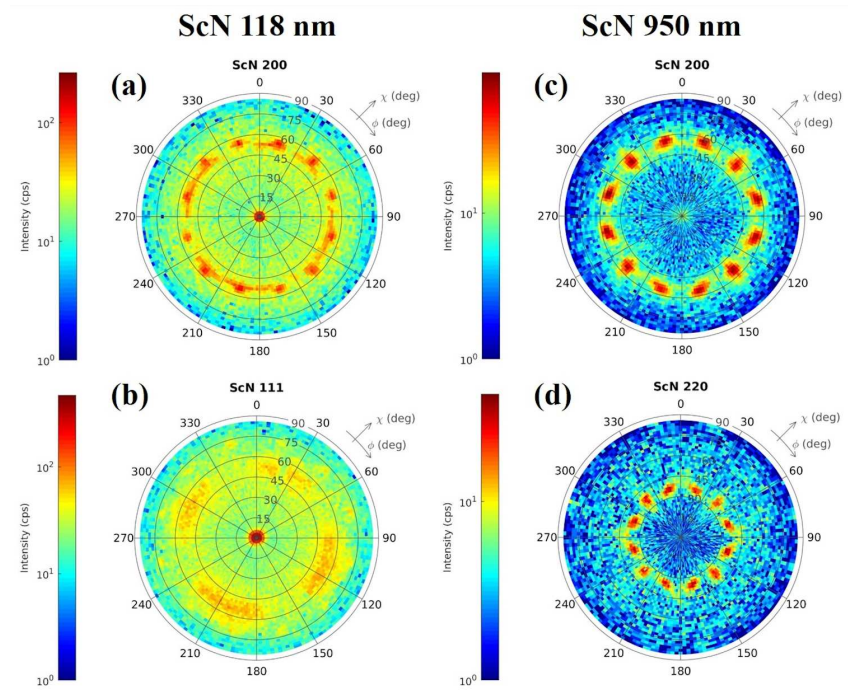

(b)

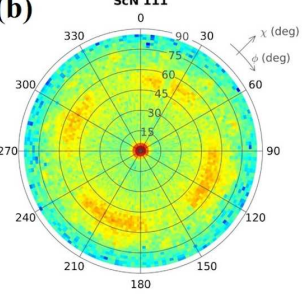

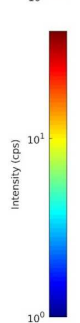

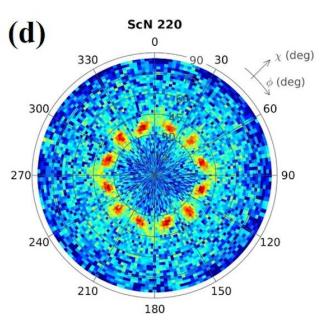

Fig. 2. Pole figures of the (200) reflection (a) and (111) reflection (b) for the $\mathrm{ScN}$ film with thickness of $118 \mathrm{~nm}$, together with pole figures of the (200) reflection (c) and (220) reflection (d) for the ScN film with thickness of $950 \mathrm{~nm}$.

orientations. A dominant (111) orientation is observed for the thicker $\mathrm{ScN}$ film of $950 \mathrm{~nm}$.

The poly-orientation is commonly observed in $\mathrm{ScN}$ thin films $[1,16]$. The ScN films usually begin to grow by epitaxial growth on a $\mathrm{MgO}$ (001) substrate with the (002) orientation of the ScN layer which subsequently changes into the (111) orientation. The formation of this mound structure is explained by a change in the adatom mobility on the surface affected by the presence of different defects during the film growth. The limitation is due to the Ehrlich-Schwoebel barrier which favors the uphill migration on terraces $[18,19]$. Figure 2 presents the pole figures of both films measured by XRD. Figure 2a and b corresponds to the (002) and (111) pole figures of $118 \mathrm{~nm}$ thick ScN film and Fig. 2c and d corresponds to the (002) and (220) pole figures of $950 \mathrm{~nm}$ thick ScN film.

The ScN film with thickness of $118 \mathrm{~nm}$ grows first by epitaxial growth in the (002) orientation with a high lateral misorientation between grains observed in Fig. $2 \mathrm{~b}$ as the presence of four diffuse (111) peaks at $\chi \approx 55^{\circ}$. 


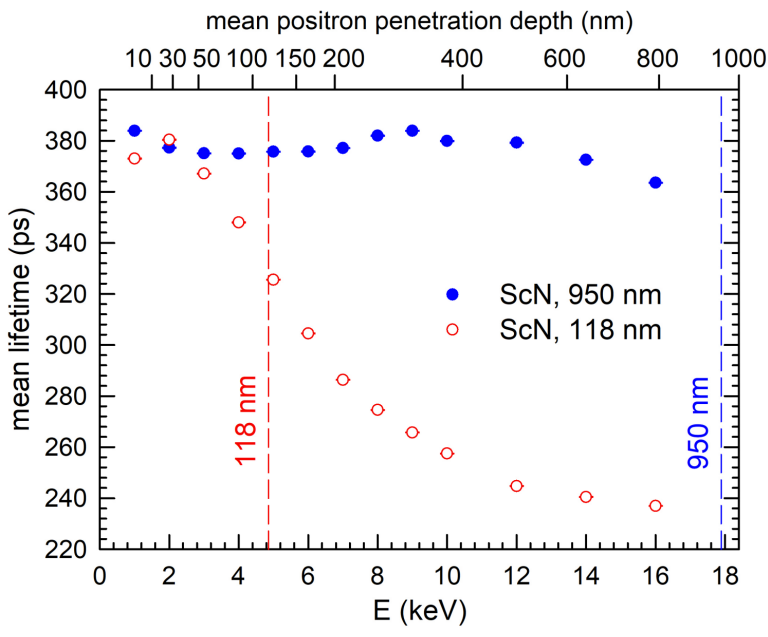

Fig. 3. The dependence of the mean positron lifetime on the energy $E$ of incident positrons. The mean positron penetration depth is indicated on the upper horizontal axis. Dashed vertical lines indicate thicknesses of both films.

Subsequently its growing mode changes to the (111) orientation demonstrated by a central peak in Fig. 2b. The pole figure of $\mathrm{ScN}$ (002) reflection shown in Fig. 2a exhibits a central peak corresponding to the $\mathrm{ScN}$ (002) epitaxial phase, and in addition 12 peaks at $\chi \approx 52^{\circ}$ coming from grains with (111) orientation. These 12 peaks correspond to twin domains grown in the $\mathrm{ScN}$ (111) grains. Considerable intensity between the 12 peaks observed in Fig. 2a indicates mosaicity between grains.

For the ScN layer with thickness of $950 \mathrm{~nm}$, both pole figures for (002) and (220) reflection shown in Fig. 2c and $\mathrm{d}$ present 12 well-defined peaks at $\chi \approx 55^{\circ}$ and $\chi \approx 35^{\circ}$, respectively. Similarly to the thinner case these peaks correspond to twin domains growth. A similar result has been reported for the formation of twin domains in $\mathrm{ScN}$ (111) films deposited on $\mathrm{MgO}$ (111) substrates [16]. The twin domains formation is induced by the nature of substrates, the deposition temperature, and the impurities which are connected to the adatom mobility during the growth process. In case of the reposted ScN film with thickness of $950 \mathrm{~nm}$, the deposition temperature of $700^{\circ} \mathrm{C}$ explains the formation of twin domains observed in the (111) oriented $\mathrm{ScN}$ phase.

Figure 3 shows the development of the mean positron lifetime with energy $E$ of incident positrons. The mean positron lifetime is a robust parameter only weakly affected by mutual correlations among the fitting parameters. For these reasons the development of the mean positron lifetime provides a useful hind of the defect depth profile of the sample studied. At low energies $(E \approx 1 \mathrm{keV})$ the majority of positrons annihilates on the surface state which is characterized by the mean positron lifetime around $380 \mathrm{ps}$ in both films. With increasing energy positrons penetrate deeper and deeper into the sample and the fraction of positrons diffusing back to the surface gradually decreases while the

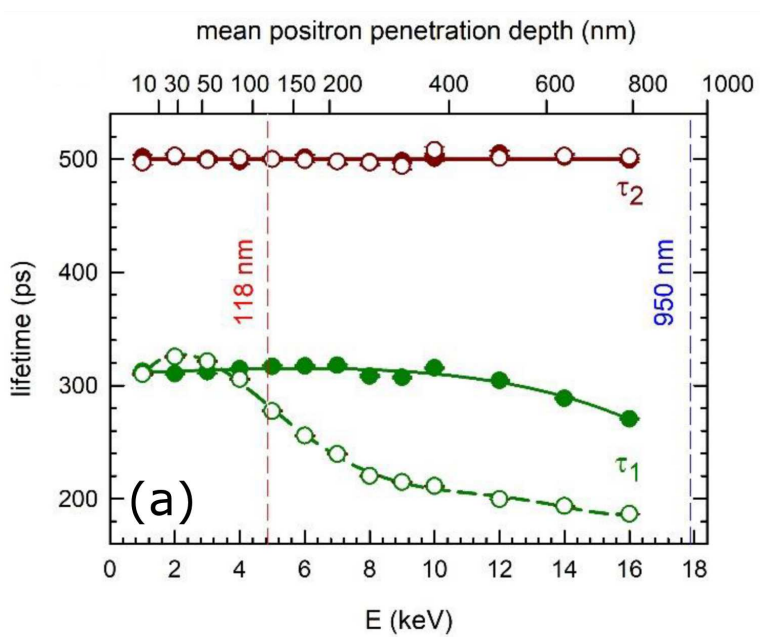

mean positron penetration depth $(\mathrm{nm})$

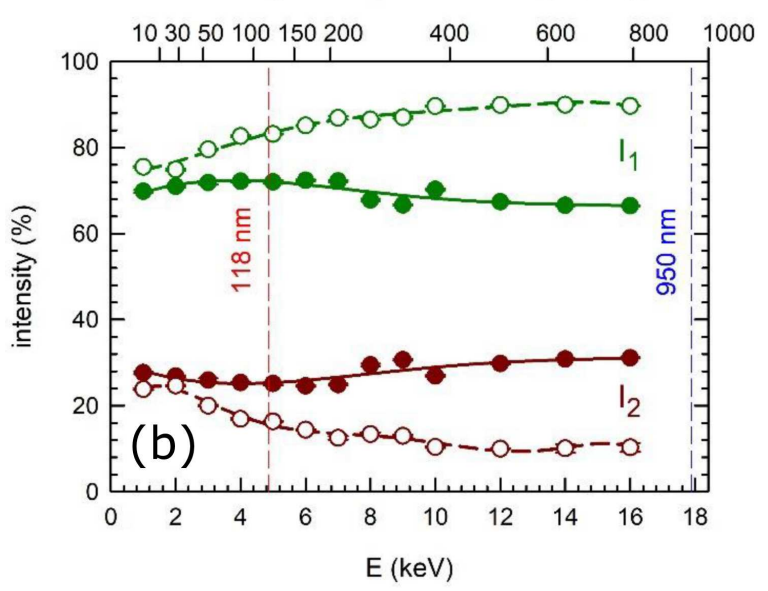

Fig. 4. The development of lifetimes (a) and relative intensities (b) of exponential components originating from positron annihilations. The mean positron penetration depth is indicated on the upper horizontal axis. Dashed vertical lines indicate thicknesses of both films. Results for the ScN film $950 \mathrm{~nm}$ are plotted by full symbols, while results for the ScN film $118 \mathrm{~nm}$ are plotted by open symbols.

fraction of positrons annihilating in the $\mathrm{ScN}$ layer increases. The mean positron lifetime for both $\mathrm{ScN}$ films is similar to the surface state. With further increase in energy positrons start to penetrate into the $\mathrm{MgO}$ substrate which is reflected by a pronounced decrease of the mean positron lifetime. This decrease occurs in the thinner film $(\mathrm{ScN} 118 \mathrm{~nm})$ already at $E \geq 3 \mathrm{keV}$, while in the thicker $(\mathrm{ScN} 950 \mathrm{~nm})$ film it was observed at $E \geq 14 \mathrm{keV}$.

More information can be obtained from decomposition of positron lifetime spectra into individual components. In addition to exponential components originating from positrons annihilating as particles, the spectra contain also a long-lived contribution which can be attributed to pick-off annihilation of ortho-positronium (o-Ps). Formation of positronium testifies the presence of nanoscopic pores in both $\mathrm{ScN}$ films. Positronium is localized in these nanoscopic pores. 


\section{TABLE I}

Calculated positron lifetimes for free positrons in a perfect $\mathrm{ScN}$ lattice (bulk) and positrons trapped at various defects. ScN with $\mathrm{NaCl}$ type structure (space group $F m 3 m$ ) and lattice parameter $a=4.505 \AA$ was considered in calculations. Calculated positron binding energies $E_{B}$ to various defects are listed in the table as well.

\begin{tabular}{l|c|c}
\hline \hline Defect type & $\tau[\mathrm{ps}]$ & $E_{B}[\mathrm{eV}]$ \\
\hline experiment & $310-325$ & - \\
bulk & 121.3 & - \\
\hline $\mathrm{V}_{\mathrm{Sc}}$ & 197.2 & 4.02 \\
$\mathrm{~V}_{\mathrm{N}}$ & 142.1 & 0.17 \\
$\mathrm{~V}_{\mathrm{Sc}}+\mathrm{V}_{\mathrm{N}}$ & 212.7 & 4.32 \\
$\mathrm{~V}_{\mathrm{Sc}}+6 \mathrm{~V}_{\mathrm{N}}$ & 276.8 & 5.35 \\
$3 \mathrm{~V}_{\mathrm{Sc}}+3 \mathrm{~V}_{\mathrm{N}}$ & 281.7 & 6.68 \\
$3 \mathrm{~V}_{\mathrm{Sc}}+7 \mathrm{~V}_{\mathrm{N}}$ & 305.3 & 6.93 \\
$3 \mathrm{~V}_{\mathrm{Sc}}+13 \mathrm{~V}_{\mathrm{N}}$ & 313.1 & 7.01 \\
$4 \mathrm{~V}_{\mathrm{Sc}}+4 \mathrm{~V}_{\mathrm{N}}$ & 327.7 & 7.67
\end{tabular}

The development of lifetimes and relative intensities of exponential components originating from positrons annihilated as particles is shown in Fig. 4a and b, respectively. Two components were resolved in positron lifetime spectra: (i) a shorter component with lifetime $\tau_{1}$ which falls into the range $310-325 \mathrm{ps}$ inside $\mathrm{ScN}$ films and decreases when positrons start to penetrate into the $\mathrm{MgO}$ substrate and (ii) a long-lived component with lifetime $\tau_{2} \approx 500 \mathrm{ps}$.

$A b$ initio calculations of positron lifetimes for various positron states in $\mathrm{ScN}$ with face-centered cubic rocksalt $(\mathrm{NaCl})$ structure with a lattice constant of $4.505 \AA$ were performed in order to link the observed lifetimes with defects in ScN films. Positron lifetimes were calculated using so-called standard scheme described in Ref. [20].

Table I shows calculated positron lifetimes for a perfect (defect-free) ScN lattice (bulk) and various point defects in $\mathrm{ScN}$. Lifetimes determined for $\mathrm{ScN}$ films in experiment are much higher than the calculated bulk $\mathrm{ScN}$ positron lifetime of 121 ps. Hence, the films contain open volume defects and their concentration is so high that virtually all positrons are trapped at defects (saturated positron trapping). Ab initio calculations showed that both scandium vacancy $\left(\mathrm{V}_{\mathrm{Sc}}\right)$ and nitrogen vacancy $\left(\mathrm{V}_{\mathrm{N}}\right)$ are capable of positron trapping. $\mathrm{V}_{\mathrm{Sc}}$ is significantly deeper positron trap characterized by a longer positron lifetime and a higher positron binding energy compared to $\mathrm{V}_{\mathrm{N}}$. The lifetime $\tau_{1}=310-325$ ps observed in the experiment is clearly higher than lifetimes of positrons trapped in $\mathrm{V}_{\mathrm{N}}$ or $\mathrm{V}_{\mathrm{Sc}}$. Hence, the component with lifetime $\tau_{1}$ comes from positrons trapped at clusters containing multiple vacancies.

Figure 5 shows calculated positron lifetimes for various point defects in $\mathrm{ScN}$ plotted as a function of their open volume. From inspection of the figure we can conclude that the lifetime $\tau_{1}$ observed in the experiment can be attributed to positrons trapped at vacancy clusters consisting mainly of $3 \mathrm{~V}_{\mathrm{Sc}}+13 \mathrm{~V}_{\mathrm{N}}$ complexes, i.e., vacancy clusters consisting of $3 \mathrm{~V}_{\mathrm{Sc}}$ and $13 \mathrm{~V}_{\mathrm{N}}$ in the nearest neighbor

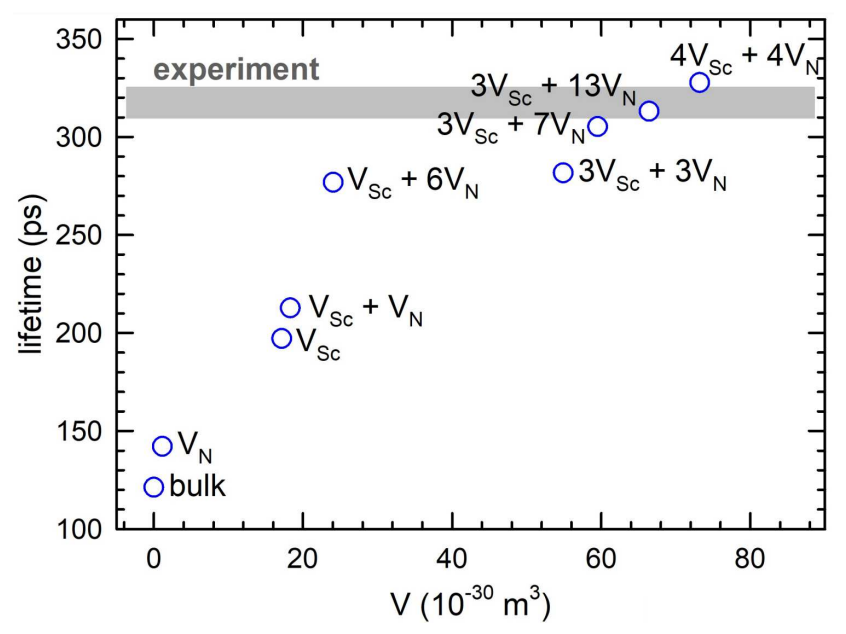

Fig. 5. Calculated lifetimes of positrons trapped at various point defects in $\mathrm{ScN}$ plotted as a function of defect open volume $V$ calculated as a sum of atomic volumes of corresponding number of Sc and $\mathrm{N}$ atoms, $V=\frac{4}{3} \pi\left(n_{\mathrm{Sc}} r_{\mathrm{Sc}}^{3}+n_{\mathrm{N}} r_{\mathrm{N}}^{3}\right)$, where $n_{\mathrm{Sc}}$ and $n_{\mathrm{N}}$ is the number of $\mathrm{V}_{\mathrm{Sc}}$ and $\mathrm{V}_{\mathrm{N}}$ in the cluster and $r_{\mathrm{Sc}}, r_{\mathrm{N}}$ denotes the atomic radius of $\mathrm{Sc}$ and $\mathrm{N}$, respectively. Gray band shows the range of the lifetime $\tau_{2}$ observed in the experiment.

positions. The calculated lifetimes for $3 \mathrm{~V}_{\mathrm{Sc}}+7 \mathrm{~V}_{\mathrm{N}}$ and $4 \mathrm{~V}_{\mathrm{Sc}}+4 \mathrm{~V}_{\mathrm{N}}$ vacancy clusters are also close to the experimental value of $\tau_{1}$ as well. Hence, one can conclude that $\mathrm{ScN}$ films contain vacancy clusters consisting of 3-4 $\mathrm{V}_{\mathrm{Sc}}$ associated with 4-13 $\mathrm{V}_{\mathrm{N}}$. The long-lived component with lifetime $\tau_{2} \approx 500$ ps comes from positrons trapped in large voids or nanoscopic pores.

The positronium contribution consists of a short-lived component with a lifetime of 125 ps originating from para-positronium ( $\mathrm{p}-\mathrm{Ps}$ ) self-annihilation and a longlived component which comes from pick-off annihilation of o-Ps. The ratio of intensities of the p-Ps and the o-Ps component is 1:3. The development of the o-Ps lifetime is shown in Fig. 6a. The intensity of positronium contribution plotted in Fig. 6b is remarkably higher for the $950 \mathrm{~nm}$ thick ScN film compared to the $118 \mathrm{~nm}$ thick film. It indicates that the concentration of nanoscopic pores in the $950 \mathrm{~nm}$ thick $\mathrm{ScN}$ film is higher than in the $118 \mathrm{~nm}$ thick film. This is most probably caused by the first (002) epitaxial growth and then the change to the (111) orientation growth which leads to a gradual disappearance of nanoscopic pores by diffusion of vacancies to the surface and to the interface between the film and the substrate. Indeed, Fig. 6a shows that the o-Ps lifetime for the $118 \mathrm{~nm}$ thick $\mathrm{ScN}$ film is enhanced at the depth corresponding to the interface between the film and the substrate indicating that the biggest voids are located at the interface. The mean size (diameter) of pores estimated from the o-Ps lifetime using the Tao-Eldrup model is $\approx 1.6 \mathrm{~nm}$ for the $950 \mathrm{~nm}$ thick $\mathrm{ScN}$ film, while for the $118 \mathrm{~nm}$ thick $\mathrm{ScN}$ film it increased up to is $\approx 2 \mathrm{~nm}$ at the film-substrate interface $[21,22]$. In the case of 
mean positron penetration depth $(\mathrm{nm})$

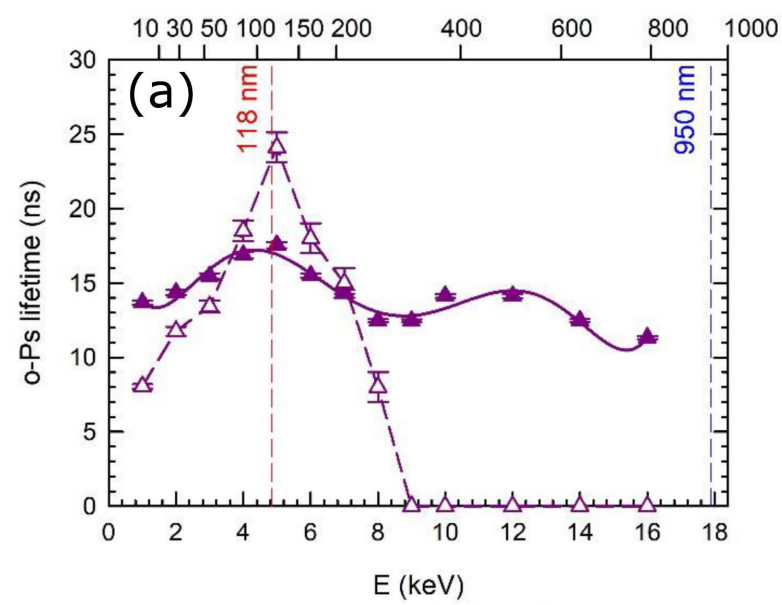

mean positron penetration depth $(\mathrm{nm})$

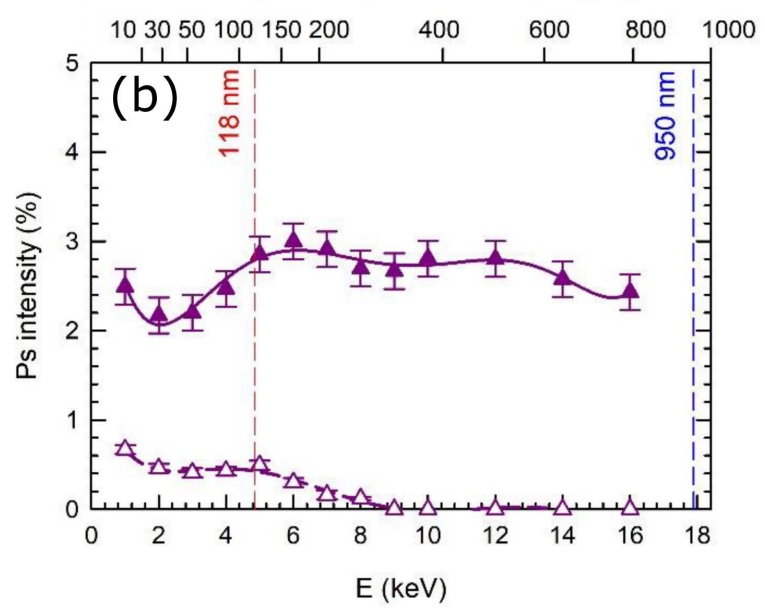

Fig. 6. The development of (a) o-Ps lifetime and (b) intensity of Ps contribution. The mean positron penetration depth is shown on the upper horizontal axis. Dashed vertical lines indicate thicknesses of both films. Results for the $950 \mathrm{~nm}$ thick $\mathrm{ScN}$ film are plotted by full symbols, while results for the $118 \mathrm{~nm}$ thick ScN film, $700{ }^{\circ} \mathrm{C}$ are plotted by open symbols.

the $118 \mathrm{~nm}$ thick $\mathrm{ScN}$ film the positronium component completely disappears for positron energies higher than $8 \mathrm{keV}$ since virtually positrons are annihilated inside the $\mathrm{MgO}$ substrate (because of small film thickness) where no positronium is formed.

The electron transport properties of $\mathrm{ScN}$ films are summarized in Table II. Resistivities of $\rho=1620$ and $86300 \mu \Omega \mathrm{cm}$, carrier concentrations of $n=6.98 \times 10^{20}$ and $2.28 \times 10^{20} \mathrm{~cm}^{-3}$, and mobilities of $\mu=5.514$ and $0.357 \mathrm{~cm}^{2} \mathrm{~V}^{-1} \mathrm{~s}^{-1}$ were measured for ScN films with thicknesses of 118 and $950 \mathrm{~nm}$, respectively. The electrical values are in good agreement with the range of values of $10-10000 \mu \Omega \mathrm{cm}$ for $\rho, 20-100 \mathrm{~cm}^{2} \mathrm{~V}^{-1} \mathrm{~s}^{-1}$ for $\mu$, and $1.1-30 \times 10^{20} \mathrm{~cm}^{-3}$ for $n$ reported for $\mathrm{ScN}$ films deposited on $\mathrm{MgO}$ by reactive sputtering $[11,23]$. Introduction of $\mathrm{V}_{\mathrm{N}}$ into ScN film tends to decrease the carrier concentration and to increase the resistivity due to the metallic
TABLE II

Electron-transport properties measured on ScN films.

\begin{tabular}{c|c|c|c}
\hline \hline $\begin{array}{c}\text { Thickness } \\
{[\mathrm{nm}]}\end{array}$ & $\begin{array}{c}\text { Resistivity } \\
\rho[\mu \Omega \mathrm{cm}]\end{array}$ & $\begin{array}{c}\text { Carrier } \\
\text { concentration } \\
n\left[\mathrm{~cm}^{-3}\right]\end{array}$ & $\begin{array}{c}\text { Carrier } \\
\text { mobility } \\
{\left[\mathrm{cm}^{2} \mathrm{~V}^{-1} \mathrm{~s}^{-1}\right]}\end{array}$ \\
\hline 118 & 1620 & $6.98 \times 10^{20}$ & 5.514 \\
950 & 86300 & $2.28 \times 10^{20}$ & 0.357
\end{tabular}

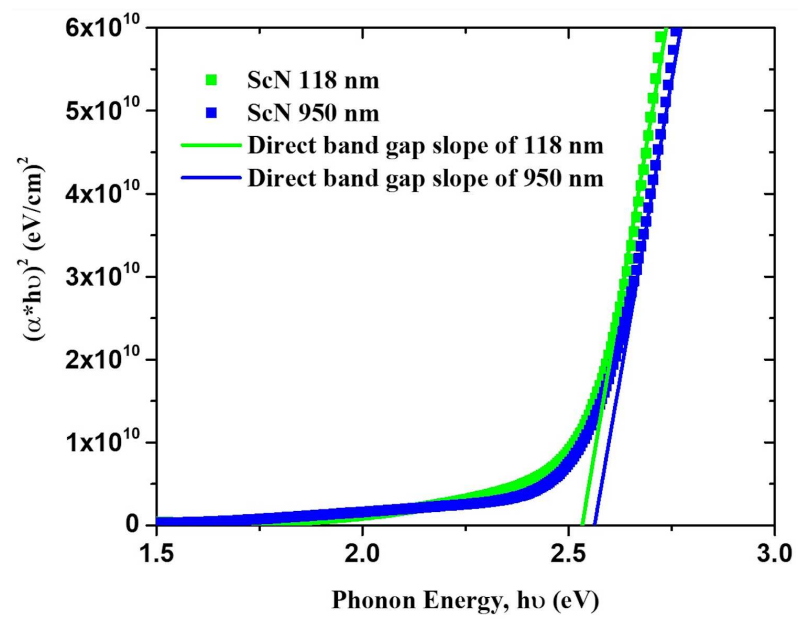

Fig. 7. Tauc's plot of ScN absorption coefficients as a function of the wavelength energy. Direct band gaps are extracted from these curves with values of $2.53 \pm 0.01$ and $2.56 \pm 0.01 \mathrm{eV}$ for $\mathrm{ScN}$ film with thickness of 118 and $950 \mathrm{~nm}$, respectively.

behavior of ScN [11]. Resistivity values measured in the $\mathrm{ScN}$ films in the present work are 10 to 100 times higher than resistivity values reported in Ref. [11] which varies from $\approx 70$ to $\approx 550 \mu \Omega \mathrm{cm}$ for the carrier concentration varying from $10.4 \times 10^{20}$ to $1.12 \times 10^{20} \mathrm{~cm}^{-3}$. This difference can be explained by the presence of vacancy clusters and less densification of our $\mathrm{ScN}$ films due to the lower temperature deposition of $700{ }^{\circ} \mathrm{C}$ compared to $\approx 900^{\circ} \mathrm{C}$ used in Ref. [11]. The temperature deposition of $700^{\circ} \mathrm{C}$ is not enough to ensure an $\mathrm{N} / \mathrm{Sc}$ ratio close to 1. A clear evolution of this $\mathrm{N} / \mathrm{Sc}$ ratio in $\mathrm{ScN}$ films deposited on $\mathrm{MgO}$ (111) substrate has been reported from 0.63 to 0.93 with the deposition temperature increasing from $700^{\circ} \mathrm{C}$ to $900{ }^{\circ} \mathrm{C}$ [16]. Vacancy clusters consisting of 3-4 $\mathrm{V}_{\mathrm{Sc}}$ associated with $4-13 \mathrm{~V}_{\mathrm{N}}$ detected in our $\mathrm{ScN}$ films contain more $\mathrm{V}_{\mathrm{N}}$ than $\mathrm{V}_{\mathrm{Sc}}$. This is in good agreement with the deposition temperature of $700^{\circ} \mathrm{C}$ when the deposition conditions can be considered as Sc rich [16]. High electrical resistivity of $\mathrm{ScN}$ films studied indicate that vacancy clusters may play a role in the reduction of the conductivity and the carrier mobility in the $\mathrm{ScN}$ films in similar way as $V_{N}$.

Figure 7 shows Tauc's plot of absorption coefficients for both $\mathrm{ScN}$ films deposited on $\mathrm{MgO}$ (001) substrate. Direct band gaps extracted from these curves are $2.53 \pm 0.01$ 
and $2.56 \pm 0.01 \mathrm{eV}$ for $\mathrm{ScN}$ films with thicknesses of 118 and $950 \mathrm{~nm}$, respectively. These values fall into the range of reported experimental values from 2.03 to $3.2 \mathrm{eV}[12,13]$. A slight difference of direct band gaps of the films with thicknesses of 118 and $950 \mathrm{~nm}$ can be caused by different carrier concentration.

\section{Conclusion}

ScN films with thicknesses of 118 and $950 \mathrm{~nm}$ were deposited on $\mathrm{MgO}$ (001) substrates. Poly-oriented (001) and (111) films containing twin domains in the (111) oriented layer were obtained. Both ScN films studied contained a high concentration of open volume defects resulting in saturated positron trapping. Two kinds of defects were detected: (i) small vacancy clusters consisting of 3-4 $\mathrm{V}_{\mathrm{Sc}}$ and $4-13 \mathrm{~V}_{\mathrm{N}}$, meaning that both films were grown in Sc rich conditions resulting in under stoichiometry in nitrogen; (ii) nanoscopic pores with average size of $1.6-2 \mathrm{~nm}$. The presence of nanoscopic pores were clearly demonstrated by formation of positronium in both films. Reduction of the concentration of nanoscopic pores in the thinner $\mathrm{ScN}$ film with thickness of $118 \mathrm{~nm}$ was explained by a higher concentration of (002) oriented grains which contain fewer defects than the (111) oriented grains. Both the ScN films studied exhibited similar band gap values of 2.53-2.56 eV. A significant degradation of the electron transport properties was detected for the thicker ScN film with thickness of $950 \mathrm{~nm}$.

\section{Acknowledgments}

The project was supported by the Grant Vakuum Praha project, by Czech Science Foundation (GACR), project: 17-05770S, by MEYS project CENAM LO1409 No. CZ.02.1.01/0.0/0.0/16 013/0001406, and by ERDF project No. CZ.02.1.01/0.0/0.0/15 003/0000485. The MePS facility has partly been funded by the Federal Ministry of Education and Research (BMBF) with the grant PosiAnalyse (05K2013). The support of the ELBE team at HZDR is greatly acknowledged.

\section{References}

[1] D. Gall, I. Petrov, N. Hellgren, L. Hultman, J.E. Sundgren, J.E. Greene, J. Appl. Phys. 84, 6034 (1998).

[2] A.R. Smith, H.A.H. AL-Brithen, D.C. Ingram, D. Gall, J. Appl. Phys. 90, 1809 (2001).
[3] J.P. Dismukes, W.M. Yim, V.S. Ban, J. Cryst. Growth 13, 365 (1972).

[4] P.V. Burmistrova, J. Maassen, T. Favaloro, B. Saha, S. Salamat, Y. Rui Koh, M.S. Lundstrom, A. Shakouri, T.D. Sands, J. Appl. Phys. 113, 153704 (2013).

[5] P. Eklund, S. Kerdsongpanya, B. Alling, J. Mater. Chem. C 4, 3905 (2016).

[6] D. Gall, M. Städele, K. Järrendahl, I. Petrov, P. Desjardins, R.T. Haasch, T.Y. Lee, J.E. Greene, Phys. Rev. B 63, (2001).

[7] M.A. Moram, Z.H. Barber, C.J. Humphreys, Thin Solid Films 516, 8569 (2008).

[8] G. Travaglini, F. Marabelli, R. Monnier, E. Kaldis, P. Wachter, Phys. Rev. B 34, 3876 (1986).

[9] A. Qteish, P. Rinke, M. Scheffler, J. Neugebauer, Phys. Rev. B 74, (2006).

[10] B. Saha, J. Acharya, T.D. Sands, U.V. Waghmare, J. Appl. Phys. 107, 033715 (2010).

[11] R. Deng, B.D. Ozsdolay, P.Y. Zheng, S.V. Khare, D. Gall, Phys. Rev. B 91, (2015).

[12] J. More-Chevalier, S. Cichoň, J. Bulír, M. Poupon, P. Hubík, L. Fekete, J. Lančok, AIP Adv. 9, 015317 (2019).

[13] B. Biswas, B. Saha, Phys. Rev. Mater. 3, 020301 (2019).

[14] H.A.H. AL-Brithen, E.M. Trifan, D.C. Ingram, A.R. Smith, D. Gall, J. Cryst. Growth 242, 345 (2002).

[15] S. Kerdsongpanya, N. Van Nong, N. Pryds, et al., Appl. Phys. Lett. 99, 232113 (2011).

[16] A. le Febvrier, N. Tureson, G. Greczynski, P. Eklund, J. Phys. D: Appl. Phys. 52, 035302 (2018).

[17] A. Wagner, M. Butterling, M.O. Liedke, K. Potzger, R. Krause-Rehberg, AIP Conf. Proc. 1970, 040003 (2018).

[18] S.C. Wang, G. Ehrlich, Phys. Rev. Lett. 70, 41 (1993).

[19] A. Gölzhäuser, G. Ehrlich, Phys. Rev. Lett. 77, 1334 (1996).

[20] M.J. Puska, R.M. Nieminen, Rev. Mod. Phys. 66, 841 (1994).

[21] S.J. Tao, J. Chem. Phys. 56, 5499 (1972).

[22] M. Eldrup, D. Lightbody, J.N. Sherwood, Chem. Phys. 63, 51 (1981).

[23] T. Ohgaki, K. Watanabe, Y. Adachi, I. Sakaguchi, S. Hishita, N. Ohashi, H. Haneda, J. Appl. Phys. 114, 093704 (2013). 